\title{
Spirulina platensis's phycocyanobilin as an antiangiogenesis by inhibiting VEGFR2-VEGFA pathway in breast cancer: in silico study
}

\author{
Dewa Ayu Putu Ismartati Sukma Jayanti' ${ }^{1}$ I Gede Agni Marwan Abimanyu' ${ }^{1}$, Haidar Azzamudin1 ${ }^{1}$ \\ ${ }^{1}$ Department of Biology, Faculty of Mathematics and Natural Sciences, Brawijaya University, Jalan Veteran, Malang, East Java, \\ Indonesia, 65145
}

Submission: 21 May 2021; Revised: 21 September 2021; Accepted: 26 September 2021

*Corresponding author: Dewa Ayu Putu Ismartati Sukma Jayanti; e-mail: sukmajayanti083@student.ub.ac.id ; tel.: 087754199427

\begin{abstract}
Breast cancer is a cancer type that leads to many women deaths. The causes are from the primary tumour and other progressions such as metastatic and angiogenesis. Some therapy strategies have been developed to treat breast cancer, but they are not good enough for treating breast cancer progressions. Spirulina platensis has a phycocyanin and a phycocyanobilin, known as antioxidant and antiinflammatory bioactivities. This study identified anticancer activity of phycocyanobilin from Spirulina platensis. We also investigated the phycocyanobilin mechanism in breast cancer inhibition through VEGFR2-VEGFA pathway. In silico analysis was conducted the inhibition modelling of phycocyanobilin to the VEGF-VEGFR pathway. The VEGF and VEGFR proteins were taken out from Protein Data Bank (PDB) database and were prepared with BIOVIA Discovery Studio 2019. Phycocyanobilin as a ligand was obtained from PubChem and prepared with PyRx. The molecular docking was conducted using HEX 8.0.0 CUDA and the last step is the protein-ligand complexes were visualized and analyzed using BIOVIA Discovery Studio 2019. It results in five protein-ligand complexes in which the receptor-ligand complex VEGFR2-[VEGFA-phycocyanobilin] can inhibit the angiogenesis process by phycocyanobilin binds to VEGFA, and it prevents the angiogenesis process by blocks the VEGFR2 and stops VEGFA to bind with VEGFR2. Thus phycocyanobilin has potential as an anticancer agent especially in breast cancer as an antiangiogenesis.
\end{abstract}

Keywords: angiogenesis, breast cancer, phycocyanobilin, Spirulina platensis, VEGFA, VEGFR2

\section{INTRODUCTION}

Breast cancer is the most common type of cancer and is the leading cause of cancer deaths in women. The primary tumor itself causes these deaths and as a result, another tumour progresses in other organs throughout the body [1]. World heatlh organization reported the incident case of breast cancer in women were 2,088,849 (24.2\%) out of 8,622,539 cancer cases at 2018 [2]. According to Indonesia's Ministry of Health INFODATIN's data in 2019 shows that the prevalence of cancer in Indonesia in women $(2.85$ $\%$ ) is greater than in men $(0.74 \%$ ) in 2018 which indicates an increase in prevalence in 2013 (women $=2.2 \%$, men $=0.6 \%$ ). Those data was supported by the Dharmais Cancer Hospital in 2018 which shows that the highest number of cancer cases is breast cancer (19.18\%) and based on the sex of the person with cancer, breast cancer is mostly suffered by women (34.3\%) [3].

Breast cancer emerges from impaired regulation of some signalling pathways that occurs in mammary ephitelial cells. Activated chemokines and growth factors are signalling molecules that promote cancer progression. Molecular pathway of cancer was the interaction between signalling molecules to their receptors. Several receptors were identified in cancer signalling pathway, including epidermal growth factor receptor (EGFR), vascular endothelial growth factor receptor (VEGFR), platelet-derived growth factor receptor (PDGFR), insulin-like growth factor receptor (IGFR), and fibroblast growth factor receptor (FGFR) [4]. The processes of the formation and maintenance of new blood and lymphatic vessels in some tissues called angiogenesis and lymphangiogenesis are initiated by vascular endothelial growth factor (VEGF) and its receptor VEGFR which is secreted and plays a role main role in this process. Angiogenesis is a central factor that stimulates blood vessel growth and formation to allow the cancer cell proliferation [5]. The VEGFA or VEGF) is a secreted ligand by tomour cells and reported as a cancer biomarker [6].

Several strategies has been studied and discovered to inhibit the VEGFR-VEGF pathway in the treatment of cancer [6]. The known neoadjuvant therapy has been proven to treat the breast cancer by monoclonal antibody therapy using bevacuzimab, which is often combined with capecitabine or trastuzumab. However, Bevacuzimab could be combined with other chemotherapy agents such as taxanes, docetaxel and paclitaxel. But, the combination performs poor inhibition of those drugs, and the redundancy 
of VEGF in cancer tissue produces other cancer factors [7] that can lower the efficacy of the therapies. Natural sources such as Spirulina sp. including Spirulina platensis has been proved as an anticancer agents from its phycocyanin which inhibits the growth and proliferation of cancer by cell cycle arrest mechanism and its pro-apoptotic activity by activating caspase- 3 and caspase- 9 $[8,9]$. Phycocyanobilin was found inside the phycocyanin as its chromopores [10]. Spirulina platensis has been known as an anticancer alternative therapy, but the study about the phycocyanobilin's bioactivity for inhibiting the angiogenesis process during the breast cancer progression is still limited. This study aims to study and understand the mechanism of phycocyanobilin from Spirulina platensis's phycocyanin in inhibiting VEGFR-VEGF pathway, which will lead to inhibition of angiogenesis process in the breast cancer progression.

\section{RESEARCH METHODS}

\section{Protein Preparation}

The protein molecules used in this study, VEGFR2 (PDB ID: 1VR2) [11] and VEGFA (PDB ID: 4KZN) [12] obtained from RCSB PDB. Those proteins were prepared by removed their water and ligand molecules surrounding them using BIOVIA Discovery Studio 2019 [13].

\section{Ligand Preparation}

Phycocyanobilin (CID: 365902) was obtained from PubChem database [14]. The ligand was prepared by minimizing the molecule energy using PyRx.

\section{Molecular Docking and Visualization}

Ligand and protein were docked using HEX 8.0.0 CUDA [15] with the "Shape+Electro" molecular docking method. The results was visualized and the protein-ligand bonds analyzed using BIOVIA Discovery Studio 2019 [13].

\section{RESULTS AND DISCUSSIONS}

All molecules have been docked in HEX 8.0.0 CUDA. They have five different protein-ligand complex combinations that have each binding interaction with their distinctive binding category, as shown in Figure 1. The receptor complex VEGFR2-phycocyanobilin has nine binding interactions consisting of a hydrogen bond and eight hydrophobic interactions resulting in binding energy at $-322.70 \mathrm{kcal} / \mathrm{mol}$, performing the highest binding energy (Table 1). The VEGFA-phycocyanobilin has a tighter interaction with lower binding energy than VEGFR2 phycocyanobilin, generating binding energy $343.92 \mathrm{kcal} / \mathrm{mol}$ with seven binding interactions, including three hydrogen bonds, an electrostatic bond, and three hydrophobic interactions.

The native protein-ligand complex VEGFR2VEGFA produces $-662.95 \mathrm{kcal} / \mathrm{mol}$ with twenty binding interactions. Ten of the interactions are hydrogen bonds. VEGFR2 shows a unique interaction - VEGFA binding, a sulfur-x bond from sulfur from Met1072 - VEGFR2 to the oxygen, nitrogen, and sulfur molecules in Glu93 of VEGFA. While the VEGFR2-phycocyanobilin receptor complex interacts with VEGFA, the binding energy of the interaction gets stronger that can be known from its binding energy is increased from $-662.95 \mathrm{kcal} / \mathrm{mol}$ in VEGFR2-VEGFA complex to $-674.74 \mathrm{kcal} / \mathrm{mol}$. Still, unfortunately, this receptor complex-ligand interaction fails to inhibit the VEGFA as the interaction doesn't show any changes in binding interactions in the receptor complex-ligand compared to the receptor complex itself. The interaction of VEGFR2 when it binds to VEGFA-phycocyanobilin showed four hydrogen bonds, an electrostatic bond, and four hydrophobic interactions, performing -704.37 $\mathrm{kcal} / \mathrm{mol}$. Vascular endothelial growth factor (VEGF) is the major pro-angiogenic factor, regulates endothelial cell growth and proliferation, increases the blood vessel's permeability, and is secreted by the tumour cell. The most active VEGF isoform, VEGFA, induced angiogenesis by activating VEGFR2 in endothelial cells [16]. VEGFA was elevated in hypoxia conditions by HIF-1A pathway [17, 18]. The HIF-1A/VEGF pathway was activated by mTOR in PI3K/Akt pathway [19]. The PI3K molecule triggered the activation of the tyrosine kinase receptor [20]. Hyperactivation of tyrosine kinase upregulates the PI3K/AKT/mTOR pathway. Activated AKT mediated cell growth and cell survival. In the same condition, TSC 1 and TSC 2 promoted Rheb GTPase to activate mTORC1 that promotes S6K. Furthermore, SREBP1 and PPAR $\gamma$ were switched on. The ULK1 was blocked to inhibit the autophagy process. mTORC2 directly activates AKT phosphorylation at Ser-473, while mTORC1 and $\mathrm{mTORC} 2$ received the signal transmission from some growth factors. This signalling promoted many cancer progressions [21]. VEGFR is the receptor tyrosine kinase that binds to VEGF, containing seven immunoglobulin-like segments, a short membrane-spanning region, and a cytosolic domain with tyrosine kinase activity. VEGFR2 is important in the angiogenesis process, especially its activity for mitosis in endothelial cells. Its kinase domain has a function to bind to other protein in signal transduction of angiogenesis, which is involved in tumor growth and metastasis. This domain consists of the $\mathrm{N}$ terminal lobe (residues 820-920) [22]. 
VEGFR2-phycocyanobilin
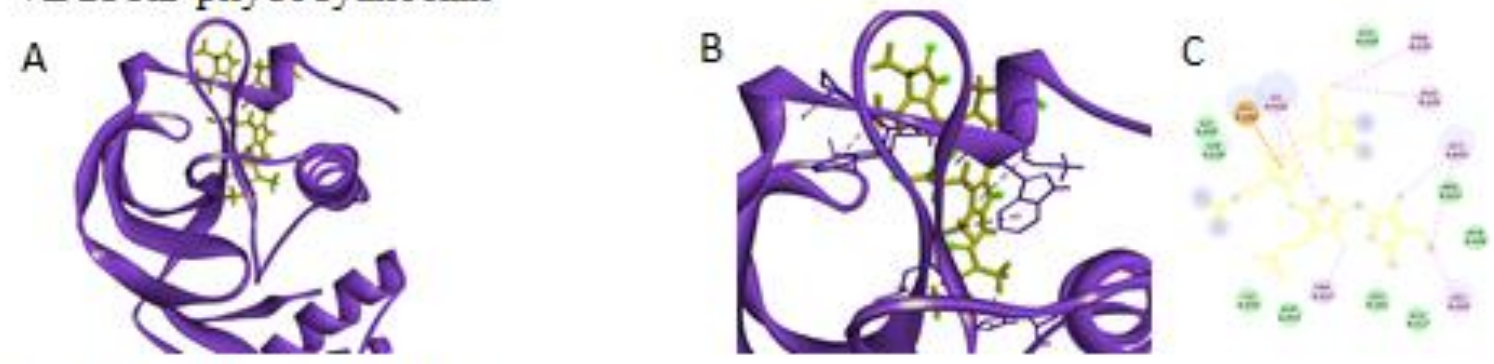

\section{VEGFA-phycocyanobilin}
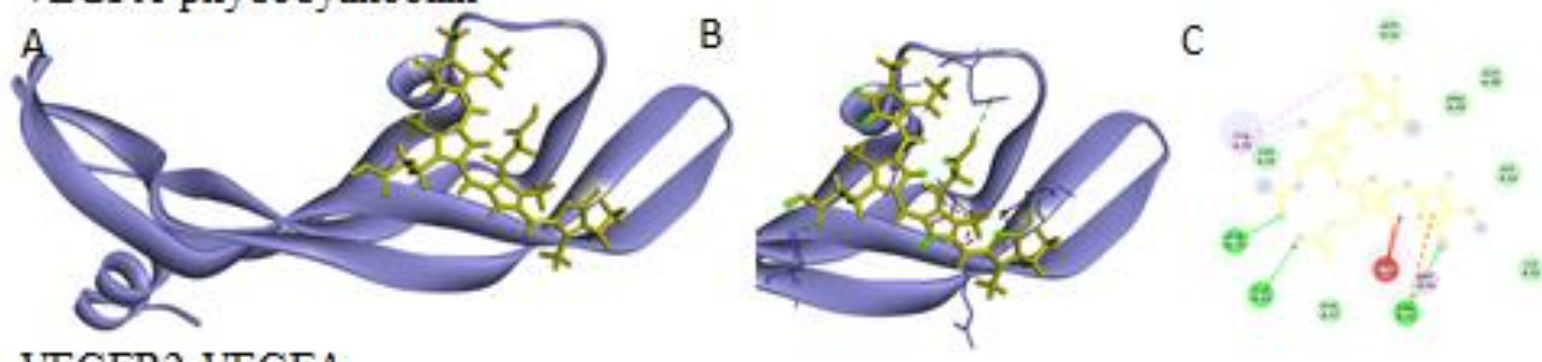

\section{VEGFR2-VEGFA}
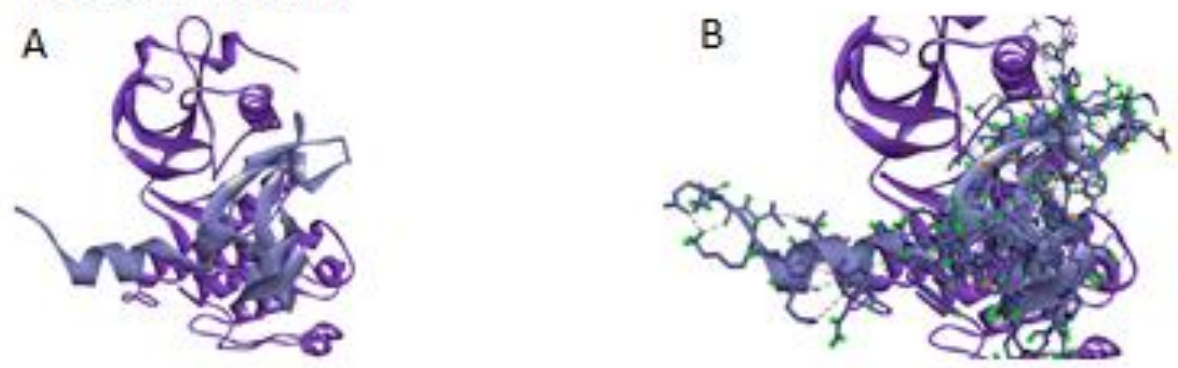

[VEGFR2-phycocyanobilin]-VEGFA

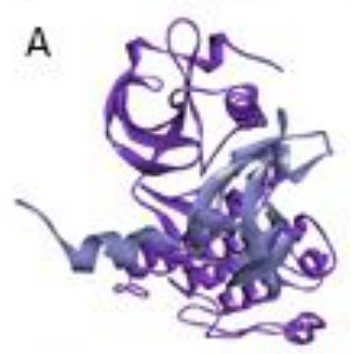

B

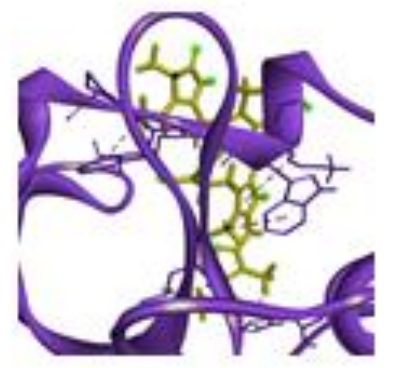

C

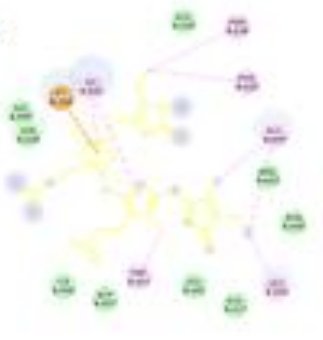

\section{VEGFR2-[VEGFA-phycocyanobilin]}

A

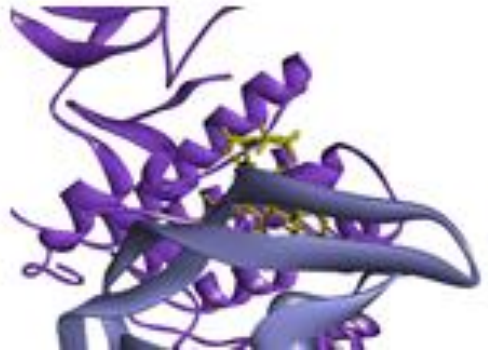

B

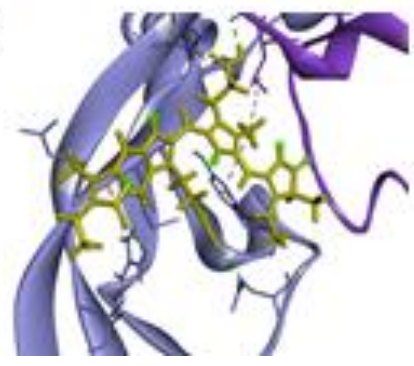

$\mathrm{C}$

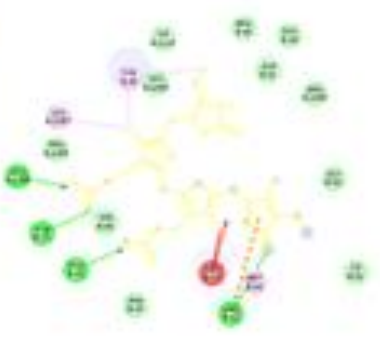

Figure 1. The binding pose of VEGFA, VEGFR2, and phycocyanobilin. Three-dimensional view of the interaction was illustrated in A-B. while, 2D performance of the interaction in C. purple color was described as VEGFR2 light purple is VEGFA, and yellow color is phycocyanobilin.

$\mathrm{N}$-terminal domain creates a fold into a twisted $\beta$ sheet with an $\alpha \mathrm{C}$ in which the $\beta$ sheet has five antiparallel strands $(\beta 1-\beta 5)$. The $\beta 1-\beta 3$ were highly curved over the $\beta 4$ and $\beta 5$. The larger C-terminal domain (residues 921-1168) contains $\beta 7$ and $\beta 8$ antiparallel strands on the top of the $\mathrm{C}$ terminal domain adjacent to the $\mathrm{N}$-terminal $\beta$ sheet. The $\alpha$ helices $(\alpha \mathrm{D}, \alpha \mathrm{E}, \alpha \mathrm{E}-\mathrm{F}, \alpha \mathrm{F}, \alpha \mathrm{G}, \alpha \mathrm{H}$, and $\alpha \mathrm{I}$ ) form the remaining core of the $\mathrm{C}$-terminal domain [22]. 
Table 1. The detail interactions among VEGFR2, VEGFA, and phycocyanobilin

\begin{tabular}{|c|c|}
\hline $\begin{array}{l}\text { Protein - ligand complexes } \\
\text { (binding energy in kcal/mol) }\end{array}$ & Binding sites residues \\
\hline \multirow{2}{*}{$\begin{array}{l}\text { VEGFR2-phycocyanobilin } \\
(-322.70)\end{array}$} & Hydrogen Bond (LYS858) \\
\hline & $\begin{array}{l}\text { Hydrophobic Interactions : } \\
\text { PRO830, LEU902, TRP827, PHE829, HIS894, LYS826 }\end{array}$ \\
\hline \multirow{3}{*}{$\begin{array}{l}\text { VEGFA-phycocyanobilin } \\
(-343.92)\end{array}$} & Hydrogen Bond : ASN75, ARG82, GLU42 \\
\hline & Electrostatic: ARG82 \\
\hline & Hydrophobic Interactions: MET94, TYR39 \\
\hline \multirow[t]{3}{*}{ VEGFR2-VEGFA (-662.95) } & $\begin{array}{l}\text { Hydrogen Bond: B:LYS48 - A:GLU885; A:ARG1032 - B:GLN79; B:GLN79 } \\
\text { - A:ASP1028; B:CYS51 - A:ALA844; B:GLN89 - A:PHE1047; B:ILE91 - } \\
\text { A:HIS1026; B:GLU93 - A:TYR1082; A:LEU1069 - B:GLU93; B:HIS90 - } \\
\text { A:HIS1026 }\end{array}$ \\
\hline & Other : A:MET1072 - B:GLU93 \\
\hline & $\begin{array}{l}\text { Hydrophobic Interactions : A:ALA844 - B:PRO49; A:ALA844 - B:CYS51; } \\
\text { A:ARG880 - B:ILE46; A:ALA881 - B:ILE46; A:ALA881 - B:ILE83; } \\
\text { A:ARG1027 - B:ILE91; B:HIS86 - A:LEU820; B:HIS90 - A:ILE1025; } \\
\text { B:HIS90 - A:ARG1027 }\end{array}$ \\
\hline \multirow{2}{*}{$\begin{array}{l}\text { [VEGFR2-phycocyanobilin]- } \\
\text { VEGFA (-674.74) }\end{array}$} & Hydrogen Bond: LYS858 \\
\hline & $\begin{array}{l}\text { Hydrophobic Interactions: PRO830; LEU902; TRP827; PHE829; HIS894; } \\
\text { LYS826 }\end{array}$ \\
\hline \multirow{3}{*}{$\begin{array}{l}\text { VEGFR2-[VEGFA- } \\
\text { phycocyanobilin] (-704.37) }\end{array}$} & Hydrogen Bond; ASN75; ARG82; ARG1066; GLU42 \\
\hline & Electrostatic: ARG82 \\
\hline & Hydrophobic Interactions: LEU1067; MET94; TYR39 \\
\hline
\end{tabular}

VEGFR2 has another functional important loop region, consisting of the glycine-rich nucleotide-binding loop (residues 841-846), the catalytic loop (residues 1026-1033), and the activation loop (residues 1046-1075). The activation loop of the protein kinase has a role in regulating its kinase activity [22]. Table 1 shows the VEGFR2 interacts with a complex VEGFAphycocyanobilin and the complex has two binding interactions in the activation loop of the VEGFR2 kinase domain. The Arg1066 and Leu1067 residues formed a hydrogen bond between the residue of the receptor and the ligand complex. Compared to the receptor complex-ligand structure, this receptor-ligand complex structure among VEGFR2, VEGFA, and phycocyanobilin is more effective in inhibiting the VEGFR2 protein kinase activity the ligand complex has bonded to the two residues in the activation loop of the protein kinase. Phycocyanobilin inhibited VEGFR2 kinase activity by preventing VEGFA VEGFR2 interaction.

The bind are formed between Arg1066 to VEGFR2 is a hydrogen bond and the Leu1067 forms a hydrophobic interaction while it has formed from the carbon element of phycocyanobilin. Both those residues are placed in the MgAMP-PNP-peptide-IRKP complex, which has a role for substrate-binding function in the VEGFR2 kinase domain [22]. The hydrogen bond that binds from the hydrogen element of phycocyanobilin to $\operatorname{Arg} 1066$ makes the bond from residues alpha-helix structure to the ligand is stable $[23,24]$. The hydrophobic interactions minimized the disturbance to energetically favourable interactions on the protein-ligand complex by self-associating the complex from the water molecules [23] and instead will associate with other hydrophobic amino acids [24]. The hydrophobic interaction also keeps the folded protein structure stable in the water [25].

\section{CONCLUSION}

Phycocyanobilin from phycocyanin of Spirulina platensis potentially as anticancer through VEGFR-VEGF inhibition pathway. Further molecular dynamic are required for the next research.

\section{ACKNOWLEDGEMENT}

We would like thank to bioinformatics assistants and lecturers for giving us tutors and teaching us the 
basics of molecular docking and other bioinformatics methods.

\section{REFERENCES}

[1] Scully, O. J., Bay, B-H., Yip, G., Yu, Y. 2012. Breast cancer metastasis. Cancer Genomics Proteomics 9(5): 311-320

[2] WHO. 2020. WHO Report on Cancer Setting Priorities: Investing Wisely and Providing Care for All. World Health Organization. Switzerland

[3] Kemenkes RI. 2019. InfoDATIN Pusat Data dan Informasi Kementerian Kesehatan RI. Kementerian Kesehatan Republik Indonesia. Jakarta Selatan

[4] Butti, R., Das, S., Gunasekaran, V. P., Yadav, A. S., Kumar, D., Kundu, G. C. 2018. Receptor tyrosine kinases (RTKs) in breast cancer: signaling, therapeutic implications and challenges. Molecular Cancer 17: 1-18

[5] Carmeliet, P. 2005. VEGF as a key mediator of angiogenesis in cancer. Oncology 69(3): 410

[6] Apte, R. S., Chen, D. S., Ferrara, N. 2019. VEGF in Signalling and Disease: Beyond Discovery and Development. Cell 176(6): 1248-1264

[7] Sledge, Jr., G. W. 2005. VEGF-Targeting Therapy for Breast Cancer. J Mammary Gland Biol Neoplasia 10: 319-323

[8] Jiang, L., Wang, Y., Yin, Q., Liu, G., Liu, H., Huang, Y., Li, B. 2017. Phycocyanin: A Potential Drug for Cancer Treatment. Journal of Cancer 8: 3416-3429

[9] Braune, S., Krüger-Genge, A., Kammerer, S., Jung, F., Küpper, J-H. 2021. Phycocyanin from Arthrospira platensis as Potential AntiCancer Drug: Review of In Vitro and In Vivo Studies. Life 11: 1-14

[10] McCarty, M. F. 2007. Clinical Potential of Spirulina as a Source of Phycocyanobilin. $J$ Med Food 10(4): 566-570

[11] https://www.rcsb.org/structure/1VR2. Accessed on Septermber 16 ${ }^{\text {th }}, 2021$

[12] https://www.rcsb.org/structure/4KZN. Accessed on September $16^{\text {th }}, 2021$

[13] https://www.3ds.com/productsservices/biovia/. Accessed on May $23^{\text {rd }}, 2021$

[14] https://pubchem.ncbi.nlm.nih.gov/compound/ 365902. Accessed on September 16 ${ }^{\text {th }}, 2021$

[15] http://hex.loria.fr/dist/index.php. Accessed on April 26 ${ }^{\text {th }}, 2021$

[16] Zhang, Q., Lu, S., Li, T., Yu, L., Zhang, Y., Zeng, H., Qian, X., Bi, J., Lin, Y. 2019. ACE2 inhibits breast cancer angiogenesis via suppressing the VEGFA/VEGFR2/ERK pathway. Journal of Experimental \& Clinical Cancer Research 38: 1-12
[17] Claesson-Welsh, L., Welsh, M. 2013. VEGFA and tumour angiogenesis. Journal of Internal Medicine 273: 114-127

[18] Kieran, M. W., Kalluri, R., Cho, Y-J. 2012. The VEGF Pathway in Cancer and Disease: Responses, Resistance, and the Path Forward. Cold Spring Harb Perspect Med 2: 1-17

[19] Li, Y., Sun, R., Zou, J., Ying, Y., Luo, Z. 2019. Dual Roles of the AMP-Activated Protein Kinase Pathway in Angiogenesis. Cells 8: 1-16

[20] Porta, C., Paglino, C., Mosca, A. 2014. Targeting PI3K/Akt $/ \mathrm{mTOR}$ signaling in cancer. Frontiers in Oncology 4(64): 1-11

[21] Ersahin, T., Tuncbag, N., Cetin-Atalay, R. 2015. PI3K/AKT/mTOR Interactive Pathway. Mol. BioSyst.: 1-9

[22] McTigue, M. A., Wickersham, J. A., Pinko, C., Showalter, R. E., Parast, C. V., Tempcyzk-Russell, A., Gehring, M. R., Mroczkowski, B., Kan, C-C., Villafranca, J. E., Appelt, K. 1999. Crystal structure of the kinase domain of human vascular endothelial growth factor receptor 2: a key enzyme in angiogenesis. Structure 7(3): 319-330

[23] Rodwell, V. W., Bender, D. A., Botham, K. M., Kennelly, P. J., Weil, P. A. 2018. Harper's Illustrated Biochemistry ThirtyFirst Edition. McGraw-Hill Education. United States of America

[24] Ferrier, D. R. 2014. Lippincott's Illustrated Reviews: Biochemistry Sixth Edition. Wolters Kluwer Lippincott Williams \& Wilkins. United States of America.

[25] Meyer, E. E., Rosenberg, K. J., Israelachvili, J. 2006. Recent progress in understanding hydrophobic 